\title{
A New Tightening Method of Bolt/Nut Assembly to Control the Clamping Force
}

\author{
Shinji HASHIMURA ${ }^{* *}$ Kyoichi KOMATSU ${ }^{* * *}$ \\ Chihiro INOUE ${ }^{* * * *}$ and Tetsuya NAKAO ${ }^{* *}$ \\ **Kurume National College of Technology, \\ 1-1-1 Komorino, Kurume, Fukuoka, 830-8555, Japan \\ E-mail: hasimura@kurume-nct.ac.jp \\ ***Tohnichi MFG. Co., Ltd., \\ 2-2-12 Omori-kita, Ota-ku, Tokyo, 143-0016, Japan \\ ****Advanced Engineering School Kurume National College of Technology,
}

\begin{abstract}
The clamping force of a bolt/nut assembly greatly influences its strength. However it is well-known that the clamping force of a bolt/nut assembly tightened by the torque control method varies widely. In this study, a new tightening method of a bolt/nut assembly that controls the clamping force more accurately has been proposed. In the proposed method, a bolt/nut assembly is tightened by pulling the bolt thread portion protruding through the nut with a force corresponding to the target clamping force. Experiments to verify the usefulness of the proposed method have been performed. The results show that the proposed method can accurately tighten a bolt/nut assembly with an error of approximately $\pm 10 \%$ in relation to the target.
\end{abstract}

Key words: Bolt/Nut Assembly, Clamping Force, Tightening Method, Bolt, Nut

\section{Introduction}

Bolt clamping force greatly influences the performance of a bolt/nut assembly. An error in the clamping force often causes loosening and fatigue failure of the bolt/nut assembly in service ${ }^{(1-2)}$. Bolts and nuts are generally tightened by the torque control method. The torque control method can tighten a bolt/nut assembly more easily than other methods. However the clamping force of bolt/nut assemblies which have been tightened by the torque control method varies widely from $\pm 20 \%$ to $\pm 50 \%$ in relation to the target clamping force ${ }^{(3-4)}$. Therefore it is important to develop a new tightening method to control the clamping force more accurately.

A new tightening method to control the clamping force of bolt/nut assemblies more accurately has been proposed. In the proposed method, a bolt/nut assembly is tightened by pulling the bolt thread portion protruding through a nut with a force corresponding to the target clamping force while the reaction force due to the pulling force is supported at the upper surface of the nut. Experiments have been performed in this study to verify the validity of the proposed method.

\section{Theory}

Figure 1 shows a schematic illustration of a bolt/nut assembly. The bolt/nut assembly consists of a bolt, a nut, clamped part I and clamped part II as

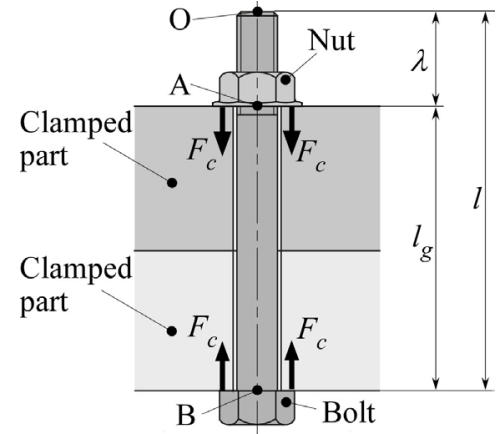

Fig.1 A bolt/nut assembly 
shown in Fig.1. In the bolt/nut assembly, the clamping force $F_{c}$ is applied to the bolt as tensile force and is applied to the clamped parts as compressive force due to tightening. In reality, the clamping force $F_{c}$ is distributed to each bolt thread engaging with the nut. However for convenience it is assumed that $F_{c}$ is concentrated at the first bolt thread shown as Point A. In Fig. 1, $l$ is the nominal length of the bolt. Points O, A and B are point on the axis running through the center of the bolt. Point $O$ is at the bolt tip, point $A$ is a point at the base of the nut. Point $\mathrm{B}$ is a point corresponding to the bolt neck. $\lambda$ is the distance between point $\mathrm{O}$ and point $\mathrm{A} . l_{g}$ is the grip length.

In the proposed method, the bolt/nut assembly is tightened by three main tightening processes. Fig. 2 shows schematic illustrations of the three processes. In Process I, the bolt thread portion protruding through the nut is pulled with pulling force $P$ corresponding to target clamping force $F_{c}$ while the reaction force is supported at the upper surface of the nut. For convenience, the pulling point is defined as point $\mathrm{O}$ in this paper. When point $\mathrm{O}$ is pulled by $P$, the portion of $\lambda$ on the bolt is stretched. During this process, the bolt thread surface in contact with the nut thread surface is opposite to the situation where the bolt is actually tightened.

Fig. 3 shows behaviors of some parameters in Processes II and III. Fig. 3(a) shows the behavior of the distance $\lambda$ between point $\mathrm{O}$ and point $\mathrm{A}$ versus the rotation angle $\theta$ of the nut if the pulling force $P$ is maintained. Fig. 3(b) shows behaviors of the pulling force $P$ and the clamping force $F$ of the bolt/nut assembly versus the rotation angle $\theta$ of the nut if the length of $\lambda$ is maintained. Process II and Process III in Fig. 3(a) and (b) correspond to Process II and Process III in Fig. 2 respectively.

In Process II, the clamping force $F$ is generated for the bolt/nut assembly by tightening the nut while maintaining $P$. The bolt portion of $\lambda$ alone is stretched only until $F$ reaches $P$. As the nut continues to be tightened, when $F$ reaches $P$, the entire nominal length $l$ of the bolt starts to be stretched. After the bearing surface of the nut touched the upper surface of the clamped part, $\lambda$ increases in proportion to the rotation angle $\theta$ of the nut as shown in Fig. 3(a) until $F$ reaches $P$. At that moment, the increase rate of $\lambda$ drastically changes because the bearing surface of the nut receives an upward force from the clamped part. At the instant at which $F$ has reached $P$, the nut rotation is stopped, and Process II is finished.

Incidentally, when the bolt starts to be stretched along the entire nominal length, $\lambda$ increases due to bolt elongation. Let $\delta_{A}$ be the displacement of point $\mathrm{O}$ if $F$ is less than $P, \delta_{A}$ is expressed as follows.

$$
\delta_{A}=\frac{P}{C_{F A}}
$$

where $C_{F A}$ is the spring constant of the portion between point $\mathrm{O}$ and point A. Let $\delta_{B}$ be the

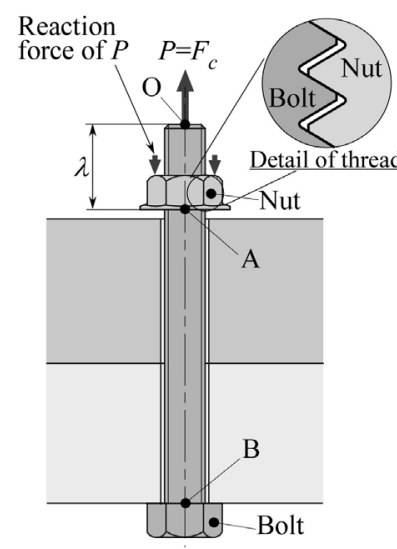

(a) Tightening process I

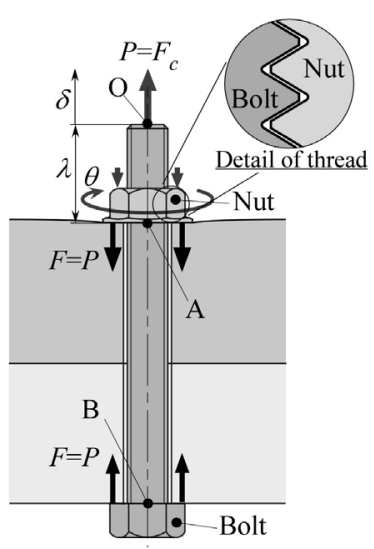

(b) Tightening process II

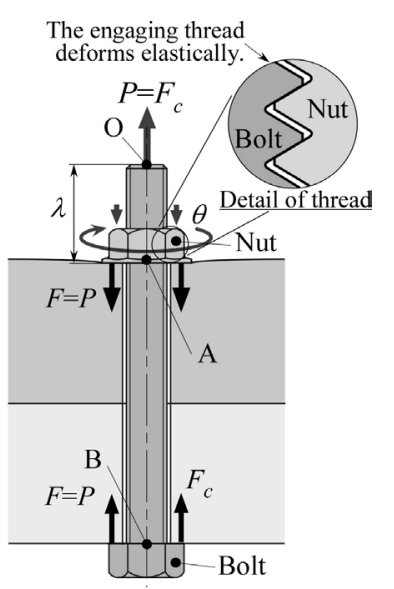

(c) Tightening process III

Fig.2 Tightening processes in the proposed method 


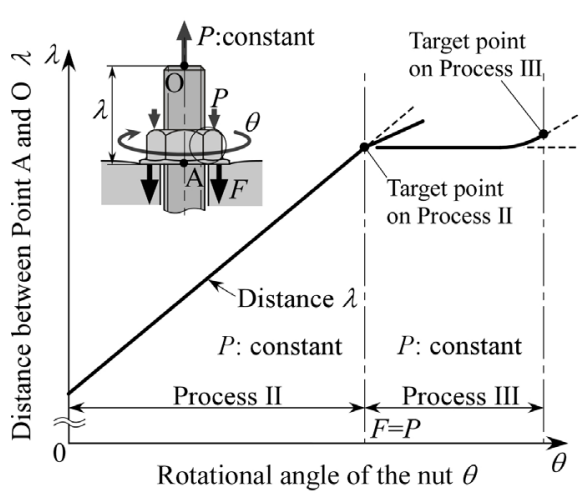

(a) Behaviors of the distance $\lambda$ if the pulling force $P$ is constant.

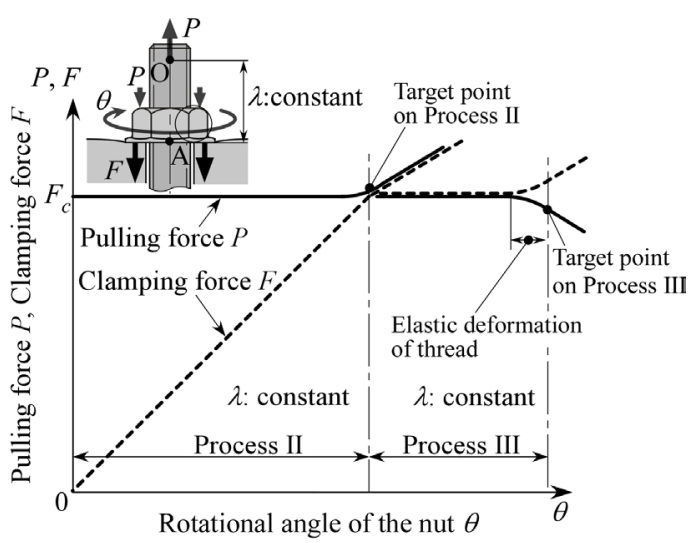

(b) Behaviors of the pulling force $P$ and the clamping force $F$ if the distance $\lambda$ is constant.

Fig.3 Variations of the pulling force and the clamping force against in each process

displacement of point $\mathrm{O}$ if $F$ is greater than $P, \delta_{B}$ is expressed as follows.

$$
\delta_{B}=\frac{P}{C_{F B}}
$$

where $C_{F B}$ is the spring constant of the portion between point $\mathrm{O}$ and B. Since $C_{F A}$ is greater than $C_{F B}, \delta_{B}$ is larger than $\delta_{A}$. Therefore, $\lambda$ becomes $\lambda+\left(\delta_{B}-\delta_{A}\right)$. However the increase rate of $\lambda$ drastically changes because the variation of $\lambda$ is larger than the elongation, $\left(\delta_{B}-\delta_{A}\right)$.

After Process II, the tightening of the bolt/nut assembly cannot be considered finish because the bolt threads and the nut threads are separating due to backlash between these threads. In Process III, the nut must be further tightened until the bolt/nut assembly reaches its actual tightened situation. After Process II, the nut is tightened while the pulling force $P$ is maintained. After the bolt threads touch the nut threads, these threads begin to slightly deform elastically. Fig.2 (c) shows the actual tightened thread situation of the bolt/nut assembly. In Process III, $\lambda$ is constant until the bolt threads touch the nut thread. After these engaging threads touched each other, $\lambda$ slowly increases at first and then begins to increase linearly because the nut is tightened as shown in Fig.3(a). Process III is stopped at the instant at which $\lambda$ starts to increase linearly. And then pulling force $P$ is unloaded, the tightening device is removed, and the tightening process is finished.

Incidentally, it is not easy to implement the theory of the proposed method. So, we have accomplished by means of maintaining the distance $\lambda$ between point A and B instead of maintaining pulling force $P$. Fig. 3(b) shows the schematic chart in the form of $F$ and $P$ versus $\theta$ in Processes II and III. The solid line shows the behavior of $P$, and the dotted line shows the behavior of $F$. In Process II of Fig. 3(b), $\lambda$ is fixed at a constant length by simultaneously letting point $\mathrm{O}$ move according to the movement of point A. In Process III, $\lambda$ is fixed at a constant length without allowing the movement of point $O$. In Process II, if the length of $\lambda$ is fixed at a constant rather than pulling force $P, P$ is constant until the clamping force $F$ reaches $P$. At that moment $P$ begins to increase. At the instant at which $F$ reaches $P$, the gradient of $F$ changes concurrently with $P$. Process II is stopped at the inflection point. In Process III, if $\lambda$ is fixed at a constant length instead of $P, P$ is constant until the engaging threads touch each other. After that, the thread surfaces deform elastically. At that time, $P$ gradually decreases and $F$ increases. After the elastic deformation of the thread is almost finished, $P$ starts to decrease linearly. Process III is stopped at the point in which $P$ starts to decrease linearly. The tightening process is completely finished when the tightening device is removed.

\section{Experiment}

Figure 4 shows the experimental apparatus. Fig. 4(a) shows the schematic experimental apparatus and Fig. 4(b) shows the tightening device and a bolt/nut assembly. 
In the apparatus, the tightening device can move in a perpendicular direction and the axis of the tightening device is rotated with a motor as shown in Fig.4 (a).

In Fig. 4(b), the internal thread portion of the tightening device and the wrench portion can rotate individually. The internal thread portion of the tightening device was engaged with the portion of the bolt thread protruding from the nut. In this device, tensile force $P$ pulling the protruded bolt thread was applied by rotating the internal thread portion. The reaction force of $P$ was supported by the upper surface of the nut. Tensile force $P$ was measured by load cell A. The nut was rotated using the wrench portion. The internal thread portion and the wrench portion could be separately and simultaneously rotated using the planetary gear system, the one-way clutch and so on.

The bolt/nut assembly consists of a bolt, a nut, clamped parts I, II and load cell B as shown in Fig.4 (b). Load cell B was a hollow cylinder with an inside diameter of $D_{i}=16 \mathrm{~mm}$ and an outside diameter of $D_{o}=24 \mathrm{~mm}$. The clamping force $F$ was measured using load cell $\mathrm{B}$ for monitoring during the experiment. The test bolt was a commercial hexagon head bolt M10. The nominal length $l$ of the bolt was $100 \mathrm{~mm}$, and the thread length $l_{b}$ was $27 \mathrm{~mm}$. The property class of the bolt was 4.8 . The test nut was a hexagon nut with a flange. The grip length of bolted joint $l_{g}$ is $75 \mathrm{~mm}$. The two clamped parts were made of medium carbon steel JIS S55C. The bearing surfaces contacting the bolt and the nut were polished with \#600 sand paper before each experiment. A washer was not used in the experiments. The threads and bearing surfaces of the bolted joint were lubricated with $\mathrm{MoS}_{2}$ grease. The target clamping force $F_{c}$ was set at $10 \mathrm{kN}$ and $15 \mathrm{kN}$. The experiments were performed five times for the each target clamping force.

In the experiment, after the tightening device was attached to the un-tightened bolt/nut assembly as shown in Fig.4 (b), the protruded bolt thread was pulled by the internal thread portion with tensile force $P$ corresponding to target clamping force $F_{c}$ while being supported on the upper surface of the nut in Process I. $P$ was applied by rotating the internal thread adaptor. In Process II, the nut had to be tightened to apply the clamping force to the bolt/nut assembly while maintaining $\lambda$. So, the internal thread portion and the wrench portion were simultaneously rotated at the same speed $\omega=5 \mathrm{rpm}$. In Process III, the nut was

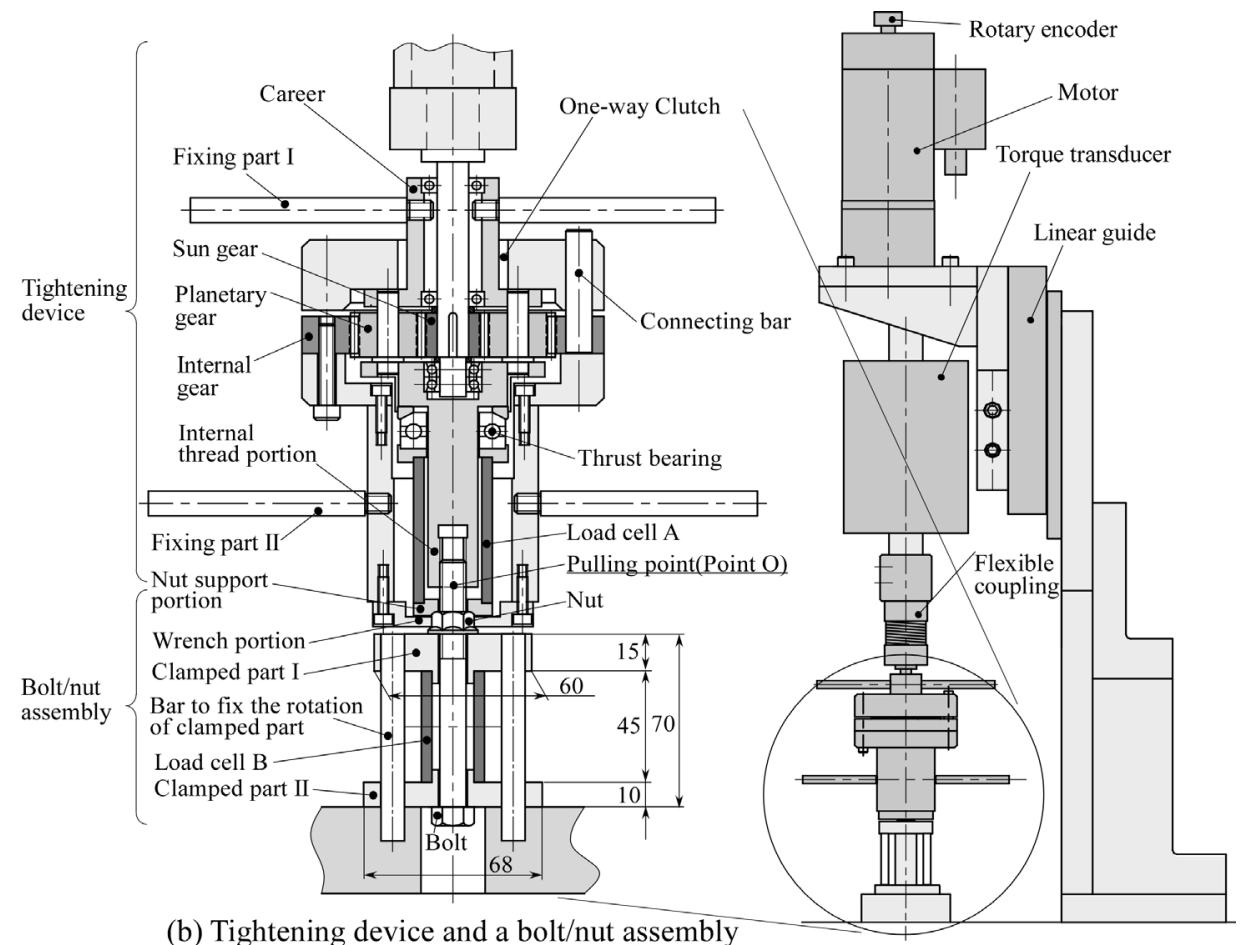

(a) A schematic experimental apparatus

Fig.4 Experimental apparatus 
tightened by rotating only the wrench portion to let the engaging pattern of the bolt thread become the same as in an actual tightening situation while maintaining $\lambda$. Then the tightening device was removed from the bolted joint. The stopping conditions of Process II and III were determined by preliminary experiments. In the preliminary experiment of Process II, the variations of $P$ and $F$ against $\theta$ were measured by tightening the nut until about $5 \mathrm{kN}$ more than the target clamping force in Process II. The stopping condition of Process II was determined from these experimental data. The stopping condition of Process III was determined using the same procedures.

\section{Experimental Results and Discussion}

Figures 5 and 6 show examples of Process II and Process III in the experimental result for $F_{c}=10 \mathrm{kN}$. Fig. 5 shows the behaviors of tensile force $P$, clamping force $F$ and gradient $d P / d \theta$ of $P$ versus rotation angle $\theta$ of the nut in Process II. Fig. 6 shows the behaviors of $P$, $F$ and $d P / d \theta$ in Process III. In Fig. 5 and 6, the left ordinates are $P$ and $F$. The right ordinate is $d P / d \theta$. The abscissa is the rotation angle $\theta$ of the nut. In Process II, the nut and the internal thread adaptor were rotated simultaneously because the nut is tightened while maintaining the distance $\lambda$ between point $\mathrm{O}$ and point A. In Process III, only the nut was rotated while maintaining $\lambda$.

As it can be seen in Fig.5, $P$ started to curve when $F$ reached $P$ which was set in target clamping force $F_{c}$. In the experiments, the condition for ending in Process II for $F_{c}=10 \mathrm{kN}$ and $F_{c}=15 \mathrm{kN}$ was determined as $d P / d \theta=0.08 \mathrm{kN} / \mathrm{deg}$ from preliminary experimental results. In this experiment, clamping force $F$ of the bolt/nut assembly in Process II was 11.5 $\mathrm{kN}$, and this was a little higher than the target. This is because of a time lag between the start times for rotating the nut and the internal thread adaptor due to the planetary gear system although the rotation of the nut and the internal thread adaptor must be begun simultaneously in order to accurately maintain $P$. In Fig. 5, $P$ increased markedly at the first stage of Process II. This is caused by the followings. At the start of Process II, the rotation of the nut and the internal thread adaptor must be simultaneously started and rotated. However since their rigidities are different, these two rotation angles have a gap. It is the gap that caused this phenomenon.

As it can be seen in Fig.6, $P$ gradually decreased inversely to the increase of clamping force $F$. This behavior can also be seen clearly from the variation of $d P / d \theta$. This is because the thread surfaces of the bolt and the nut were touching each other due to geometrical misalignment of the bolt and the nut although these surfaces theoretically do not touch each other in the first part of Process III. $P$ started to decrease significantly at around $\theta=22 \mathrm{deg}$ because the thread of the bolt and the nut made firm contact and started to deform. After

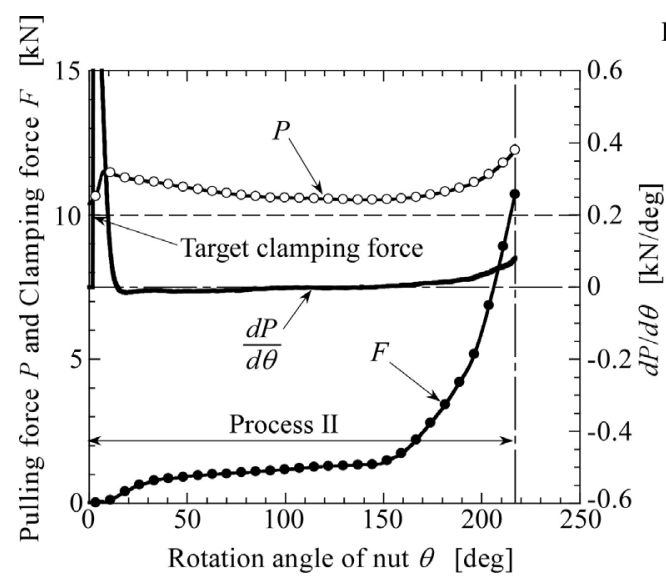

Fig. 5 Variations of the pulling force and the clamping force against the rotation angle of the nut in Process II
Decrease of $F$ after the tightening device was removed

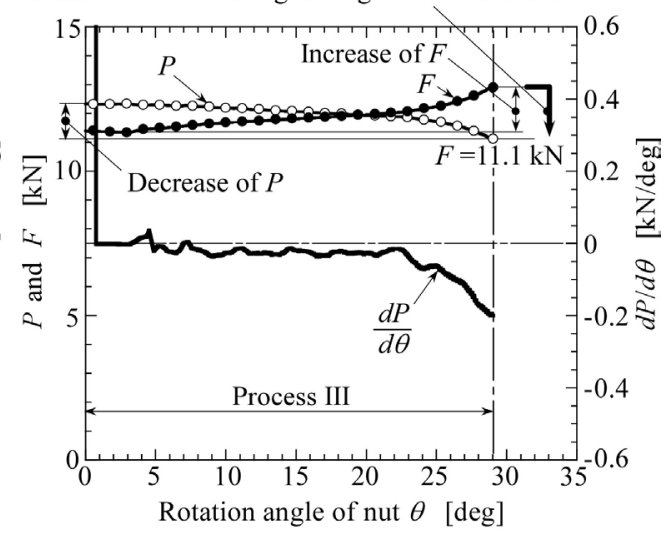

Fig.6 Variations of the pulling force and the clamping force against the rotation angle of the nut in Process III 
that, if the nut was continuously rotated, $P$ would start to decrease linearly. But, in the proposed method, the rotation of the nut was stopped because the thread situation became the actual tightening situation before $P$ started to decrease linearly. Incidentally, the condition for ending in Process III for $F_{c}=10 \mathrm{kN}$ was determined as $d P / d \theta=-0.20 \mathrm{kN} / \mathrm{deg}$ from preliminary experiments. The condition for $F_{c}=15 \mathrm{kN}$ was determined as $d P / d \theta=$ $-0.22 \mathrm{kN} / \mathrm{deg}$.

After Process III was completed, the tightening device was detached from the bolt/nut assembly. At that time, clamping force $F$ was reduced by releasing tensile force $P$. The reduction of $F$ is caused by the elastic deformation of the bolt and nut threads. The reduction of $F$ corresponds to the increase of $F$ and the decrease of $P$ in Process III because the nut is tightened while pulling the protruded bolt thread. In other words, although the increase of $F$ due to tightening the nut is added to the clamping force as an additional force, the additional force is released by detaching the tightening device. However if the nut was not tightened until the end point of Process III shown in Fig. 3, the reduction of $F$ would become greater and would not correspond to the increase of $F$ because the elastic deformation of the threads would not be sufficient. In our experiments, the final clamping force was $11.1 \mathrm{kN}$ as shown in Fig. 5 and 6. The reduction of $F$ after Process III was $2.2 \mathrm{kN}$ whereas the increase of $F$ in Process III was $1.8 \mathrm{kN}$. The difference was $0.4 \mathrm{kN}$, and it can be seen that both variations of $F$ are almost the same.

Tables 1 and 2 show the experimental results for $F_{c}=10 \mathrm{kN}$ and $F_{c}=15 \mathrm{kN}$ respectively. In these tables, the clamping force after each process, the final clamping force and the tightening error against the target are shown. It can be seen from Table 1 that the scatter band of the final tightening error was from $0 \%$ to $11.0 \%$. The scatter band of $F$ is approximately within $\pm 5 \%$ of the average although the average of $F$ is $6 \%$ larger than the target. The average of the final clamping force was $10.6 \mathrm{kN}$.

It can also be seen from Table 2 that the scatter band of the final tightening error was from $1.3 \%$ to $7.2 \%$, and this scatter band is smaller than the results for $F_{c}=10 \mathrm{kN}$. The scatter band of $F$ is approximately within $\pm 3 \%$ of the average although the average of $F$ is $4.4 \%$ larger than the target. The average of the final clamping force was $15.7 \mathrm{kN}$. We would like to consider the reason why the scatter band was reduced with an increase in the clamping force. In these results, the difference between the maximum and the minimum clamping forces was $1.1 \mathrm{kN}$ for $F_{c}=10 \mathrm{kN}$, and $0.9 \mathrm{kN}$ for $F_{c}=15 \mathrm{kN}$. Although the scatter

Table 1 Experimental results $\left(F_{c}=10 \mathrm{kN}\right)$

\begin{tabular}{c|c|c|c|c|c}
\hline \hline $\begin{array}{c}\text { Experimental } \\
\text { No. }\end{array}$ & $\begin{array}{c}F \text { after } \\
\text { Process I } \\
{[\mathrm{kN}]}\end{array}$ & $\begin{array}{c}F \text { after } \\
\text { Process II } \\
{[\mathrm{kN}]}\end{array}$ & $\begin{array}{c}F \text { after } \\
\text { Process } \\
\text { III }[\mathrm{kN}]\end{array}$ & $\begin{array}{c}\text { Final clamping force } \\
F[\mathrm{kN}]\end{array}$ & $\begin{array}{c}\text { Tightening } \\
\text { error [\%] }\end{array}$ \\
\hline \hline 1 & 10.1 & 11.5 & 13.3 & 11.1 & 11.0 \\
\hline 2 & 10.2 & 11.6 & 12.5 & 10.6 & 6.2 \\
\hline 3 & 10.1 & 11.3 & 13.5 & 11.0 & 10.0 \\
\hline 4 & 10.0 & 9.4 & 12.2 & 10.0 & 0.0 \\
\hline 5 & 10.1 & 11.7 & 13.3 & 10.4 & 4.0 \\
\hline \hline
\end{tabular}

Table 2 Experimental results $\left(F_{c}=15 \mathrm{kN}\right)$

\begin{tabular}{c|c|c|c|c|c}
\hline \hline $\begin{array}{c}\text { Experimental } \\
\text { No. }\end{array}$ & $\begin{array}{c}F \text { after } \\
\text { Process I } \\
{[\mathrm{kN}]}\end{array}$ & $\begin{array}{c}F \text { after } \\
\text { Process II } \\
{[\mathrm{kN}]}\end{array}$ & $\begin{array}{c}F \text { after } \\
\text { Process } \\
\text { III }[\mathrm{kN}]\end{array}$ & $\begin{array}{c}\text { Final clamping force } \\
F[\mathrm{kN}]\end{array}$ & $\begin{array}{c}\text { Tightening } \\
\text { error [\%] }\end{array}$ \\
\hline \hline 1 & 15.0 & 15.3 & 17.1 & 15.2 & 1.3 \\
\hline 2 & 15.0 & 16.5 & 17.9 & 16.1 & 7.2 \\
\hline 3 & 15.0 & 16.0 & 17.7 & 15.9 & 5.9 \\
\hline 4 & 15.1 & 16.0 & 17.9 & 15.7 & 4.9 \\
\hline 5 & 15.1 & 15.9 & 17.0 & 15.4 & 2.7 \\
\hline \hline
\end{tabular}


band for $F_{c}=10 \mathrm{kN}$ was approximately twice as large as it was for $F_{c}=15 \mathrm{kN}$, the difference between the maximum and the minimum clamping force was almost same. Therefore it can be seen that the amount of error does not depend on the target clamping force. If the target clamping force was great, the percentage of error in relation to the target would be reduced. Consequently it has been verified from these results that the proposed method can control the clamping force more accurately.

Concerning the reason that the average was higher than the target clamping force, it is considered that the increase of $P$ at the first stage of Process II is a leading cause of the error because clamping force $F$ in Process II directly influences the final clamping force. The detailed causes of this error must be discovered before the development stage.

\section{Conclusion}

The main conclusions obtained in this study are summarized as follows.

1. A new tightening method to accurately control clamping force by pulling the protruded bolt thread with a tensile force corresponding to the target clamping force has been proposed.

2. In the proposed method, the tightening error was from $0 \%$ to $11.0 \%$ for the target clamping force $10 \mathrm{kN}$. The scatter band of $F$ is approximately within $\pm 5 \%$ of the average although the average of $F$ is $6 \%$ larger than the target.

3. In addition, the tightening error was from $1.3 \%$ to $7.2 \%$ for the target clamping force 15 $\mathrm{kN}$. The scatter band of $F$ is approximately within $\pm 3 \%$ of the average although the average of $F$ is $4.4 \%$ larger than the target.

4. It was confirmed that the proposed method can accurately control the clamping force of a bolt/nut assembly.

\section{References}

(1) S. Kasei, M. Ishimura, and N. Ohashi, "On Self-loosening of Threaded Joints in the Case of Absence of Macroscopic Bearing-surface Sliding", Bulletin of Japan Society of Precision Engineering, Vol.23, pp.31-36, 1989.

(2) S. Hashimura et al., "A Measurement Method of Clamping Force of Bolted Joint", Proceedings of the International Conference on Manufacturing, Machine Design and Tribology, Seoul, Korea, June 23- 24, 2005.

(3) Japanese Standard Association, 1990, Japanese Industrial Standard B 1083.

(4) K. Mori, S. Hashimura et al., "A New Tightening Method for Bolted Joints by Simultaneous Application Torque and Compressive Force", SAE 2001 Transaction, Journal of Materials and Manufacturing, Vol.110 Section 5, pp.818-824, 2002. 